\title{
Endocannabinoid Signaling in Spermatozoa: A Mini Review
}

\author{
A. Mahajan, P. Sharma, S.J. Yadav and D.K. Swain* \\ Sperm Signalling Laboratory, Department of Veterinary Physiology, College of Veterinary Science and Animal Husbandry, U.P. \\ Pandit Deendayal Upadhayaya Pashu Chikitsa Vigyan Vishwavidyalaya Evam Go Anusandhan Sansthan, Mathura, \\ Uttar Pradesh, INDIA \\ "Corresponding author: DK Swain; E-mail: dilip_swain@yahoo.com
}

Received: 28 Feb., 2020

Revised: 12 March, 2020

Accepted: 22 March, 2020

\begin{abstract}
Spermatozoa undergoes array of signaling and intracellular pathways and ultimately become competent enough to accomplish fertilization. Hormones, ion channels and signaling molecules in both male and female reproductive tract show bidirectional cross play. The recent discovery of endocannabinoids and their receptors in male and female reproductive system opened new vistas for their research in regulating sperm function. Interestingly, endocannabinoids regulate sperm motility, capacitation, hyperactivity and eventually acrosome reaction. However, their complex intracellular pathways are still to be understood in regulating spermatozoa function. The present review highlights the major breakthrough research in the area of endocannabinoids in male reproduction and in more specific in sperm cells, and their association with regulation of sperm fertilizing competence.
\end{abstract}

Keywords: Endocannabinoid, Spermatozoa, Motility, Capacitation;Acrosome reaction

Reproductive events are controlled by variety of humoral substances secreted by the hypothalamic-pituitary-gonadal axis at different levels and their complex interplay leads to successful gametogenesis, fertilization and parturition required for normal continuation of life. Besides the central axis, a large number of humoral substances have been recently shown to play pivotal role in reproduction. These substances exhibit autocrine, paracrine, neurocrine and endocrine functions by interacting with the receptors. In the recent past, endocannabinoids have been discovered to play critical role in reproduction through complex set of signalling pathways and molecules.

Various classical studies have shown that endocannabinoids negatively control the sperm function by reducing motility, capacitation, membrane potential and hyperactivation (Miller et al., 2016). The emerging role of endocannabinoids, their ligands and receptors has fascinated reproductive biologists to explore their molecular involvement in regulating reproductive functions. Endocannabinoids have been associated with both male and female reproductive events; however, in male studies pertaining to endocannabinoids is meager and still at infancy. In the last one decade, the potential involvement of endocannabinoids in male reproduction and male reproductive process has created its own space in understanding the physiological basis of sperm function. The close association of endocannabinoids in regulating sperm motility, viability, metabolism, hyperactivity, capacitation and acrosome reaction has made more interesting to overview the mechanistic insights of endocannabinoids in regulating spermatozoa function (Meccariello et al., 2014; Battista et al., 2015). Signaling pathways and intracellular communication pathways are very complex and involves array of receptors, ion channels, signalling molecules and bio-metabolites. Employing cellular, molecular, imaging and electrophysiological interventions, extensive information have been generated regarding sperm functional dynamics. In spite of all these findings, still it is very difficult to understand the cellular and molecular basis of spermatozoa function.

How to cite this article: Mahajan, A., Sharma, P., Yadav, S.J. and Swain, D.K. (2020). Endocannabinoid signaling in spermatozoa: A mini review. J. Anim. Res., 10(2): 163-171. 
In the last decade, male infertility has been increased dramatically due to various known and unknown causes. Feeding, lifestyle and habits are now playing significant role in regulating male fertility. Fertility competence of spermatozoa is yet to be understood at molecular level as mere knowing concentration, livability and motility gives insufficient information regarding the fertilizing ability of spermatozoa.

The proposed mini-review potentially wants to highlight the key involvement of endocannabinoids in regulating spermatozoa function. The current mini-review is an attempt to gather scientific literature from various research made by various workers in the area of endocannabinoid signaling in spermatozoa and current work being carried out by our group. The review will try to address the key signaling pathways of endocannabinoids in sperm cells and will establish a possible line of future research in order to understand the role of endocannabinoids in regulating sperm fertilizing competence.

\section{The endocannabinoid system (ECS)}

The ECS comprises of group of endogenous lipids including amides, esters and ethers of long chain polyunsaturated fatty acids which bind to specific membrane cannabinoid receptors (CBRs) located in the central and peripheral nervous system (Maccarrone et al., 2005; Meccariello et al., 2014). Cannabinoid receptors are the molecular targets of $\Delta 9$-tetrahydrocannabinol (THC) which is the major psychoactive principle of cannabis. Cannabis has been used as a therapeutic and recreational drug for centuries which binds to the cannabinoid receptor 1 (CB1) and cannabinoid receptor 2 (CB2) to mediate its central and peripheral actions including modulation of mood, memory, appetite, pain, pregnancy and parturition (Maccarrone et al., 2005). More recently their role in regulation of spermatogenesis and sperm functions has been exploited (Meccariello et al., 2014; Battista et al., 2015) and their potential role in regulation of spermatozoa functions in human spermatozoa have been reported.

eCBs are produced "on demand" from the membrane phospholipids by the synthesizing machinery present in the membrane followed by their extracellular transportation leading to their physiological effects. These endocannabinoids (eCB) along with the cannabinoid receptors, the enzymes that synthesize and degrade the endocannabinoids and membrane transporters constitute the endocannabinoid system (ECS). Growing use of endocannabinoids has opened new dimensions to understand how they interact and interplay to regulate sperm function (Rossato et al., 2004; Ricci et al., 2007; Meccariello et al., 2014). Following figure depicts the basic organization of endocannabinoid system and its constituents (Adapted from Meccariello et al., 2014).

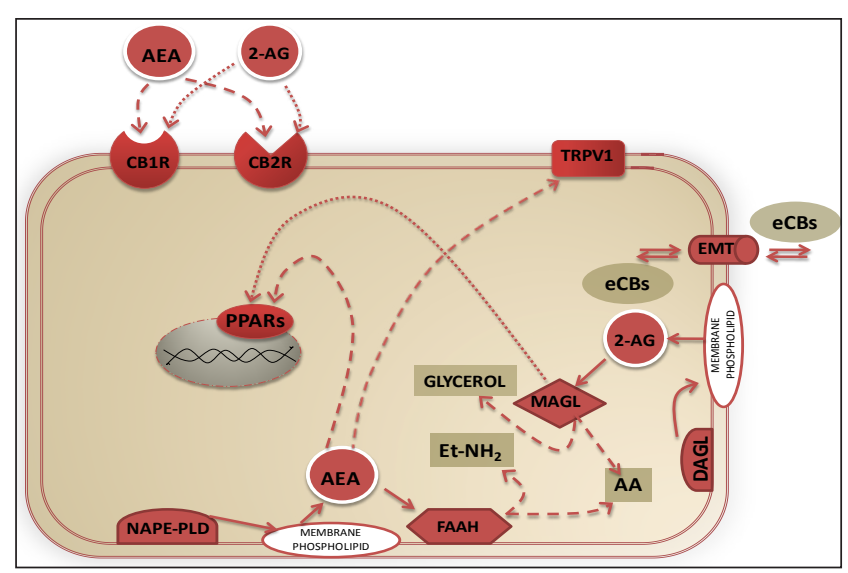

Fig 1: Overview of enocannabinoid system (ECS). Endocannabinoids (eCB) Aanandamide (AEA) and 2-Acylglycerol (2-AG) are synthesized from membrane phospholipids by the synthesizing enzymes $\mathrm{N}$-acylphosphatidyl ethanolamine hydrolyzing phospholipase-D (NAPE-PLD), Diacylglycerol lipase (DAGL); degraded by metabolic enzymes fatty acid amide hydrolase (FAAH) and monoacylglycerol lipase (MAGL) into its constituent arachidonic acid (AA), ethanolamine $(\text { Et-NH })_{2}$ and glycerol, respectively. Inside the cell, AEA binds to peroxisome proliferator activated receptor (PPARs) present on nuclear membrane and transient receptor potential vanilloid 1 (TRPV1) in the cell membrane. eCBs are transported through the endocannabinoid membrane transporter (EMT) and act on cannabinoid receptor $1(\mathrm{CB} 1 \mathrm{R})$ and cannabinoid receptor 2 (CB2R) present on the plasma membrane of the cell.

\section{Endocannabinoids and Spermatozoa}

Endocannabinoids are lipid based neurotransmitter involved in regulating cognition, appetite, pain, mood, memory, fertility, pregnancy, during pre and postnatal development, and in mediating the pharmacological effects of cannabis. They are rapidly released from the cell membrane phospholipids when stimulated by depolarizing agent (Schmid, 2000). Endocannabinoids are ligands for cannabinoid receptors derived from arachidonic acid (the unsaturated fatty acid) (Maccarrone et al., 
2005 ) and show relationship with phytocannabinoid $\Delta 9$ tetrahydrocannabinol, which is the main constituent of cannabis, by mimicking its pharmacological actions (Pertwee, 2010). Arachidonoylethanolamine (AEA, anandamide) and 2-arachidonoylglycerol are the major endocannabinoids present in the animal body while some other endocannabinoid like compounds have been discovered which activate the CBRs to show their biological activity or through "entourage effect". AEA was the first identified endogenous agonist (endocannabinoid) for cannabinoid receptors in the year 1992, followed by 2-arachidonoyl glycerol (2-AG) (Devane et al., 1992; Mechoulam et al., 1995; Sugiura et al., 1995). AEA is considered to be a novel member of the eicosanoid family (Burstein et al., 1995).

High performance liquid chromatography/mass spectroscopy has reported the presence of AEA in seminal plasma, mid- cycle oviductal fluid and follicular fluid indicating the physiological significance of AEA in regulating reproductive function (Schule et al., 2002). In order to utilize endocannabinoids such as AEA, spermatozoa should possess the biochemical machinery to synthesize, bind, transport and degrade endocannabinoids. Studies have shown the presence of type-1 cannabinoid receptors (CB1R), vanilloid receptors (TRPV1), AEAsynthesizing phospholipase D (NAPE-PLD), AEA transporter (AMT) and AEA hydrolase (FAAH) in boar spermatozoa (Maccarrone et al., 2005).

Similarly, human spermatozoa possess alpha/beta hydrolase domain containing protein 2 (ABHD2) which belongs to the superfamily of metabolic serine hydrolases; enzymes that metabolize phsopholipids and endocannabinoids. In presence of progesterone, this ABHD2 mediates the activation of CatSper by depleting the inhibitory effect of endocannabinoids (Miller et al., 2016). Using immunoblotting, fatty acid amide hydrolase (FAAH) activity in bull spermatozoa has been reported (Gervasi et al., 2009) indicating its potential role in regulating endocannabinoid signalling in sperm cells. Interestingly, AEA has been reported to act independently to $\mathrm{CB} 1$ receptors and promptly interacts with several ion channels and thereby produce resultant effects. AEA binds to T-type $\mathrm{Ca}^{++}$channels, TRPV1, voltage gated $\mathrm{K}^{+}$channels and alpha 7- nicotinic acetyl choline receptors (Van der Stelt et al., 2005) to exhibit its functional dynamics.

\section{Cannabinoid Receptors and their Signalling}

In 1990, CB1 receptors were discovered from rat cerebral cortex cDNA library and were seen to mediate the pharmacological effects of $\Delta 9$-tetrahydrocannabinol, the main psychoactive constituent of cannabis and its analogs (Matsuda et al., 1990). CB1 receptor mRNA were found mainly in the neuronal tissue and amino acid sequencing of these receptors show 97 to $99 \%$ homology in rat, mouse and human tissue. Later in 1993, CB2 receptors were identified in the human promyelocytic leukemic cell line HL60 and showed response in presence of THC and its analogs, thereby defining the receptor as being cannabinoid receptor (Munro et al., 1993). CB2 receptor mRNA were found mainly in immune system and notably absent from nervous cells. Cloned CB2 receptors from mouse showed $82 \%$ homology to human $\mathrm{CB} 2$ receptors. Both these receptors belong to the superfamily of $\mathrm{G}$ protein coupled receptors (GPCR) which mediate there action via the $G_{i / o}$ proteins, in addition to which $\mathrm{CB} 1$ receptors can signal through Gs proteins also (Pertwee et al., 2010). They act by inhibiting the adenyl cyclase and initiating mitogen activated protein kinase activity. Cannabinoid receptor associated G-proteins are linked to various sodium and potassium channels (Pertwee et al., 2010). Following figure depicts the molecular signal transduction pathways of cannabinoids (Adapted from Maccarrone, 2009).

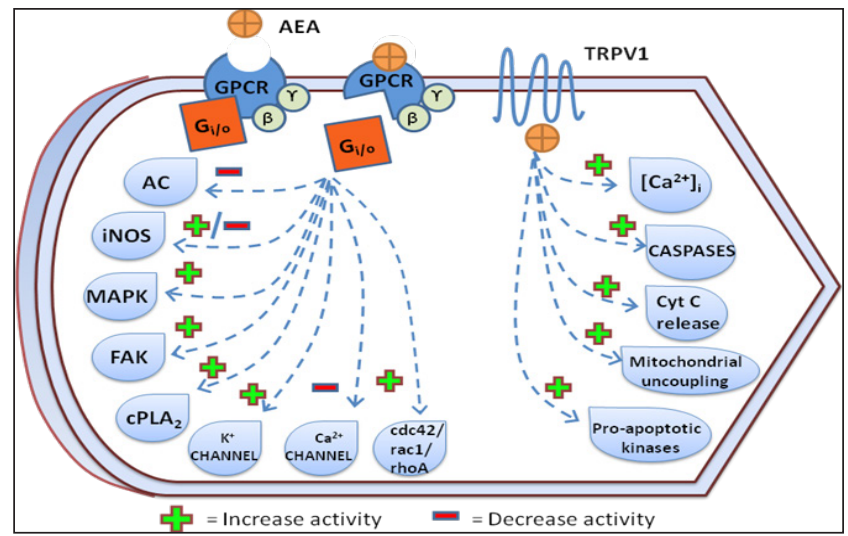

Fig. 2: Signal transduction pathways of Cannabinoids in cells. Cannabinoid receptors are G- protein coupled receptors that mediate their action via $\mathrm{G}_{\mathrm{i} / \mathrm{o}}$ protein. AEA on binding to CBRs up regulates inducible nitric oxide synthase (iNOS), mitogen activated protein kinase (MAPK), focal adhesion kinase (FAK), cytosolic phospholipase $\mathrm{A}_{2}\left(\mathrm{cPLA}_{2}\right), \mathrm{K}^{+}$channel and cdc42/rac1/ rhoA and down regulates adenyl cyclase and $\mathrm{Ca}^{2+}$ channels while on binding to the TRPV1 it increases $\mathrm{Ca}^{2+}$ influx and intercellular 
$\mathrm{Ca}^{2+}$ concentration, increase Caspase activity, cytochrome C release, mitochondrial uncoupling and pro-apoptotic changes.

Besides neuronal and immune distribution of CB1 and $\mathrm{CB} 2$ receptors, reproductive cell localizations have been reported. Studies have shown presence of both the cannabinoid receptors (CB1 and CB2) in human, bovine, porcine, mouse, amphibian and sea urchin spermatozoa as well as their male reproductive system (Francavilla et al., 2009; Maccarrone et al., 2005; Rossato et al., 2008; Gervasi et al., 2009). Immunofluorescent techniques have identified head and middle piece immunoreactivity for CB1 receptors while tail/flagellar part remained negative. Gervasi et al., (2009) identified two bands of $\sim 58 \mathrm{kDa}$ and $\sim 38 \mathrm{kDa}$ using $\mathrm{CB} 1$ and $\mathrm{CB} 2$ specific antibody in western blot analysis in bovine spermatozoa and oviductal epithelial cells, respectively. Immunohistochemistry results showed strong immunoreactivity of $\mathrm{CB} 1$ receptors in the equatorial region of head and weak immunoreactivity at the acrosome; while CB2 receptors showed positive immune reactivity at the apical region of acrosome.

\section{ECS AND MALE REPRODUCTION}

The following figure depicts the time frame of discovery of ECS in regulating male reproductive functions (Adapted from Meccariello et al., 2014).

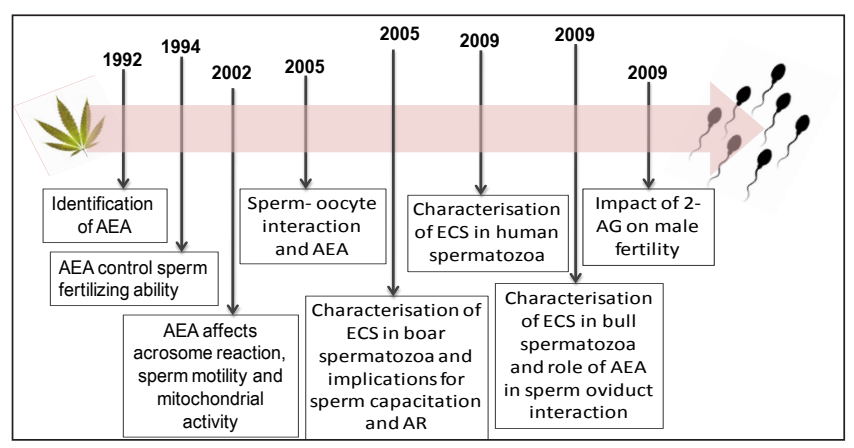

Fig. 3: Milestones in endocannabinoid signaling in male reproduction. Anandamide (AEA), an endogenous endocannabinoid was discovered in 1992 and its role in sperm fertilizing ability was established in 1994. Anandamide (AEA) potentially regulates sperm motility, acrosome reaction and mitochondrial activity was reported in 2002 and interrelationship between sperm-oocyte and AEA in 2005. In 2005 , endocannabinoid system (ECS) was reported in boar spermatozoa and its role in regulating sperm capacitation and acrosome reaction was established. Functional role of ECS in human spermatozoa was established in 2009 and in the same year, its involvement in regulation of sperm-oviduct binding was reported in bovines. Impact of 2-acyl glycerol (2-AG) on sperm functions was reported in 2009. Thereafter, studies have demonstrated the signaling mechanisms of ECS in sperm cells.

\section{Endocannabinoids and Testicular Cell Function}

Spermatogenesis is a complex process induced and maintained by the gonadotropins like follicle stimulating hormone (FSH) and luteinizing hormone (LH) in the mammalian system. Sertoli cell nourishes the growing spermatocyte and release spermatozoa into the lumen of the seminiferous tubule while Leydig cells also play their part in sperm development and maturation regulating steroid biosynthesis and secretion of hormones. These events are mediated via complex cross-talk between FSH, LH and rising evidence of involvement of endocannabinoids (Maccarrone et al., 2000). Both the cells have been shown to possess functional endocannabinoid system in samples obtained from 4- and 24- day old mice (Maccarrone et al., 2003) where Sertoli cells showed enhanced CB2 expression while Leydig cell showed enhanced CB1 expression. Positive immunoreactivity for CB1 and CB2 receptors on spermatozoa, Leydig cells and Sertoli cell are indicators of functional significance of ECS in regulating male reproductive function. Not only ECS regulates developmental stages of sperm cells but also mediates their functional development as well as acquisition of fertilizing competence of sperm cells.

A possible mechanism has been proposed for the modulation of Sertoli cell number involving the crosstalk between FSH and anandamide where anandamide has been shown to induce sertoli cell apoptosis, entering through the membrane transporter, via the TRPV1 receptors while this effect is counteracted by $\mathrm{CB} 2$ receptor (Maccarrone, 2000). FSH regulates Sertoli cell apoptosis by activating FAAH after binding to cell surface receptor (FSHR) (Rossi et al., 2007). Activation of FAAH results in endogenous breakdown of AEA to arachidonic acid (AA) and ethanolamine (Et- $\left.\mathrm{NH}_{2}\right)$. This eventually down regulates apoptosis of Sertoli cells (Miller et al., 2016). FSH has been reported to induce the gene promoter for FAAH which in turn increase the FAAH activity inside the Sertoli cell that breaks down anandamide into arachidonic acid and ethanolamine, thus inhibiting the AEA dependent Sertoli cell apoptosis in a dose dependent manner. This effect signifies that FAAH is the specific target for FSH 
inside Sertoli cell. Rossi et al., (2007) reported the role of protein kinase A and aromatase dependent signaling pathway in mediating FSH dependent inhibition of apoptosis involving FAAH activity. Following figure depicts the interplay of cannabinoids, gonadotropins and sertoli cells in testes (Adapted from Maccarrone, 2009). .

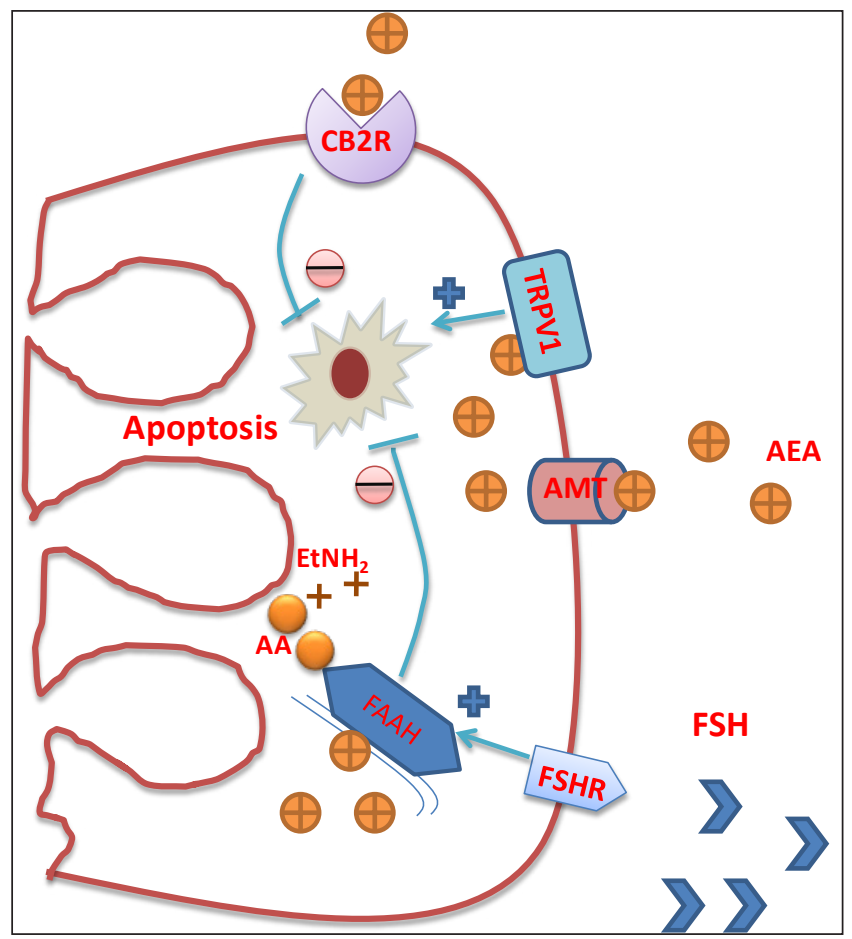

Fig. 4: Interplay between FSH, AEA and Sertoli cell function and apoptosis: Anandamide induces sertoli cell apoptosis via TRPV1 activation counteracted by activation of CB2 receptor. FSH activates FAAH which breaks down AEA into arachidonic acid (AA) and ethanolamine $\left(\mathrm{EtN}_{2}\right)$. In this way AEA mediates removal of aged sertoli cells. Endocannabinoid system regulates sertoli cell function, proliferation and apoptosis. Hormone FSH regulates sertoli cell apoptosis through endocannabinoid system. Anandamide (AEA) enters the sertoli cell through AEA membrane transporter (AMT) and after entering the cell, it binds to TRPV1 receptor, thereby it activates machinery which results in induction of apoptosis in sertoli cell. Similar to this, sertoli cell show large number of $\mathrm{CB} 2$ receptors which binds to AEA and negatively regulate apoptosis.

\section{Sperm Functional Dynamics}

Each spermatozoa is fully equipped with a functional ECS which has been shown to decrease sperm motility, capacitation induced acrosome reaction and mitochondrial activity (Rossato et al., 2004). Sperm gain the ability of hyperactivation during its transit through the female reproductive tract where it encounters different gradients of ionic concentrations and a variety of chemicals that induce the changes in the sperm membrane. Different concentrations of AEA are found in the epididymis and the female reproductive tract that put their effect on the sperm motility (Schule, 2006). Inside the male body, in the epididymis, AEA inhibit sperm motility maintaining a quiescent stage in the cauda. AEA decreases the sperm motility by binding to $\mathrm{CB} 1$ receptor resulting in inhibition of mitochondrial activity, cellular respiration and glycolysis (Battsita et al., 2015). Anandamide affect the sperm motility and viability in a dose dependent manner; at low dose, the motility decreases without affecting viability however, at high doses the viability was compromised (Rossato et al., 2004). High doses of AEA make the spermatozoa completely immotile (Rossato et al., 2004; Barbonetti et al., 2010). In sea urchin, AEA has been shown to reduce spermatozoa fertilizing capacity by modulating sperm motility (Maccarrone, 2009).

Ricci et al., (2007) reported an increase in the sperm motility in the caput epididymus of a CB1R knockout mice compared to the wild type. More recently, role of CB2 receptors in mediating sperm function has been documented, where CB2 agonists have been shown to increase the slow progressive sperm while $\mathrm{CB} 1$ agonist increases the immobile sperm cells (Agirregoitia et al., 2009). Studies have shown that AEA interacts with oxidative phosphorylation pathways and leads to inhibition of mitochondrial energy homeostasis (Barbonetti et al., 2010). Decreased mitochondrial activity is the major cause behind diminished sperm function (Rossato et al., 2004). It has been reported in boar spermatozoa that AEA reduces the capacitation and induces acrosome reaction (AR) through a cAMP pathway involving $\mathrm{CB} 1$ receptor (Maccarrone, 2005); while it inhibits the zona pellucida (ZP) induced AR via $\mathrm{CB} 1$ receptors. AEA activates the TRPV1 which reduce spontaneous AR to prevent "out of place" release of the acrosomal enzymes.

Growing evidence and hypothesis have been established that AEA regulates sperm functional dynamics by targeting the mitochondrial $\mathrm{CB} 1$ receptors. Binding of AEA to $\mathrm{CB} 1$ results in substantial reduction in mitochondrial activity leading to less consumption of energy by sperm cells and energy is reserved. When the antagonistic activities 
of CB1/AEA are withdrawn, mitochondrial machinery gets activated leading to release of bursts of energy and release of mitochondrial store of $\mathrm{Ca}^{++}$. Altogether these events lead to sperm hyperactivation, acrosome reaction and eventually fertilization (Rossato et al., 2004; Ricci et al., 2007; Meccariello et al., 2014; Battista et al., 2015).

Recently the endocannabinoids have been linked with the activation of principal calcium channel of the sperm "CatSper channel". It is established that progesterone activates the CatSper and interestingly progesterone does not bind to the CatSper directly, but in human spermatozoa, progesterone conveys its action via the endocannabinoid system by inhibiting the inhibitory effect of endocannabinoids on the CatSper (Miller et al., 2016). Thus, an increasing gradient of progesterone inside the female tract leads to activation of endocannabinoid degrading enzymes FAAH and ABHD2 which causes the inhibition of the inhibitory effect of AEA and thus promote the sperm capacitation and acrosome reaction during the terminal parts of sperm movement before fertilization. Following figure summarizes the functional involvement of endocannabinoids in regulating sperm function.

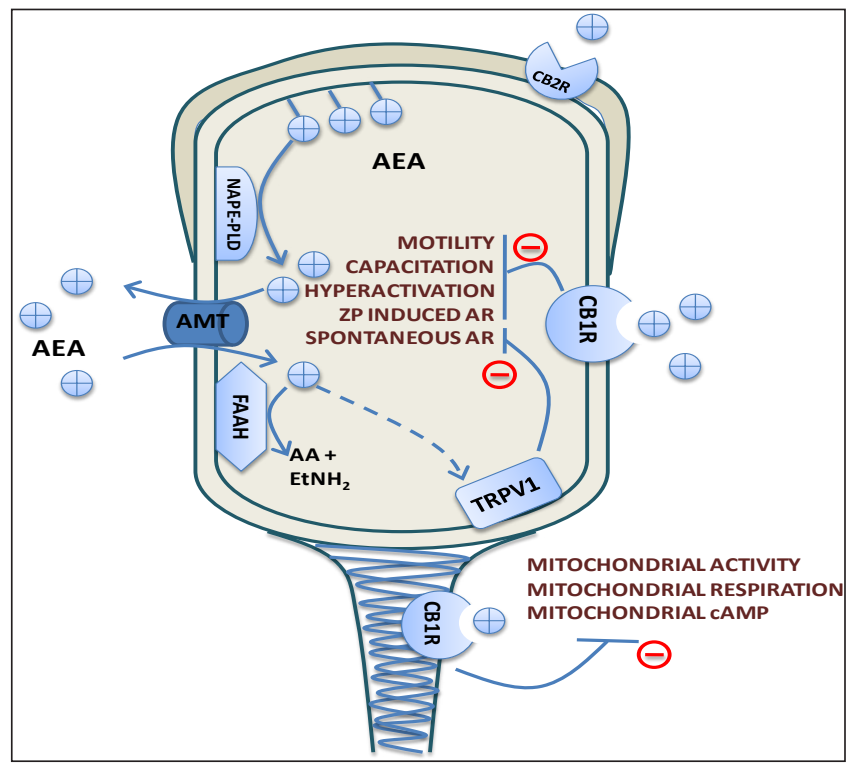

Fig. 5: Dynamic interplay between endocannabinoids and spermatozoa in regulation of sperm functions. Anandamide (AEA) is produced from the sperm membrane phospholipids by $\mathrm{N}$-acylphosphatidyl ethanolamine hydrolyzing phospholipase-D (NAPE-PLD), moves outside the cell and bind to cannabinoid receptor 1 (CB1) receptor to inhibit motility, capacitation, hyperactivation and zona pellucida ( $\mathrm{ZP})$ induced acrosome reaction (AR). Transient receptor potential vanilloid 1 (TRPV1) activation prevents spontaneous AR. Binding of AEA to CB1 receptors present on the mid piece of spermatozoa inhibits mitochondrial activity, respiration and cAMP. Fatty acid amide hydrolase (FAAH) activity is responsible for degradation of AEA into arachidonic acid (AA) and ethanolamine $\left(\mathrm{EtNH}_{2}\right)$.

\section{Sperm- Oviduct Interaction}

Spermatozoa binds to the oviductal epithelium and is released when hyperactivated (Gervasi et al., 2009). Recent reports have documented that AEA plays major role in binding and detachment of bull spermatozoa from bovine oviductal epithelium (Gervasi et al., 2009). Non hydrolysable AEA analogue metanandamide (MEA) was used to determine sperm oviduct interaction and it was found that it inhibits binding of spermatozoa to oviductal epithelium and induces it release from epithelium (Gervasi et al., 2009). Only selective CB1R antagonist was able to block effect of metanandamide while selective CB2 antagonist was not able to do so, thus indicating that only CB1R were involved in sperm oviductal epithelium interaction/binding. It has been reported that genetically defective mice with diminished FAAH activity show high concentration of AEA in the male reproductive tract thus impairing motility and these spermatozoa are not able to penetrate the zona barrier on reaching the oocyte thus leading to failure of penetration and fertilization (Sun et al., 2009). Following figure shows the different concentration gradient of anandamide (AEA) in reproductive tract and its functional dynamics with sperm cells.

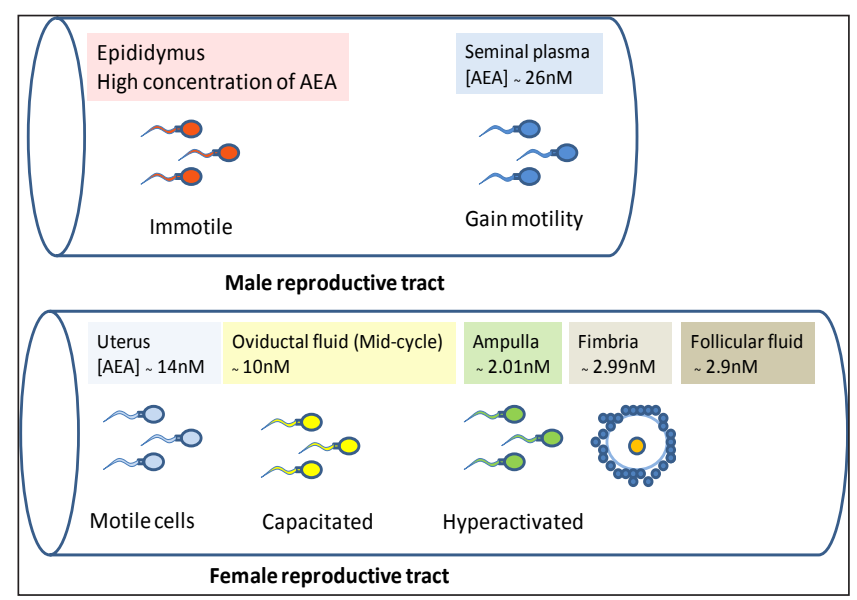

Fig. 6: Dynamics of AEA and sperm cell movement in male and female reproductive tract: Interaction of spermatozoa 
with different concentration of AEA while traversing from the male reproductive tract to the female tract. Spermatozoa face a decreasing concentration of AEA during its journey. High concentration of AEA in the cauda epididymis keeps the spermatozoa in a quiescent state while a decrease in AEA concentration in the seminal plasma imparts motility in them but no fertilizing capacity. When the spermatozoa reaches the uterus, it is still motile under high concentration of AEA. After dilution in the oviduct when the optimum concentration of AEA is there, spermatozoa get capacitated and hyperactivated thus gaining competence to reach oocyte, attach to it, release acrosomal content and penetrate the zona layer to fertilize the ova.

\section{Our Laboratory Experience}

For the last 7 years, we are in the quest to understand the role of various ion channels, hormones and effective signaling molecules in regulating sperm function. We experimented in bull and buck spermatozoa and most of the dynamic as well as kinetic studies have been performed in bovine spermatozoa. Recently, for the last two years we started evaluating the role of enocannabinoids and their receptors in regulating sperm function.

We started investigating the role of endocannabinoid system in regulating functional dynamics of bovine and caprine spermatozoa. Immunoblotting and immunofluorescence confirmed the presence of both $\mathrm{CB} 1$ and $\mathrm{CB} 2$ receptors; however, functional studies showed the potential involvement of $\mathrm{CB} 1$ receptors in regulating spermatozoa function compared to $\mathrm{CB} 2$ receptors. $\mathrm{CB} 1$ receptors were found to be dominant in regulation of sperm function. We further started investigating the non-genomic signalling of hormone progesterone $\left(\mathrm{P}_{4}\right)$ and its interrelationship with $\mathrm{CB} 1$ and $\mathrm{CB} 2$ receptors. Our preliminary findings have revealed some interesting findings like $\mathrm{P}_{4}$ non-genomic signalling is dependent on CB1 receptors and independent of CB2. We have also observed involvement of classical cAMP-PKA-PKC pathway in regulating $\mathrm{P}_{4}$ mediated nongenomic signalling and $\mathrm{CB} 1$ receptors. Inhibition of $2 \mathrm{AG}$, FAAH and NAPE modulates spermatozoa function. It is too early to further comment on this area as our studies are still incomplete and looking to the complex interplay between cannabinoid receptor and other downstream molecules, further investigations are required.

\section{CONCLUSION}

Cannabinoid receptors and their agonists and antagonists are well established and studied in the different biological systems including the reproductive system. But, very little is known about the signaling mechanism of the endocannabinoids in the spermatozoa and how they mediate their cellular actions on binding to cannabinoid receptors. The downstream pathways are still a mystery and are good area for research investigations as endocannabinoids play important role in mediating fertility in both males and females. So, it becomes a concerning topic for those who use cannabis or cannabinoid like products in both animal and human medicine. Any alteration in the eCB or the ECS components may have negative impact on the various events in fertilization and can lead to male and female infertility.

Presence of $\mathrm{CB} 1$ and $\mathrm{CB} 2$ receptors on spermatozoa invariably indicates the potential involvement of endocannabinoid in regulation of spermatozoa function. Studies in spermatozoa have already shown its involvement in energy homeostasis, capacitation and acrosome reaction; however the downstream pathways regulating $\mathrm{CB} 1$ and $\mathrm{CB} 2$ receptor function in spermatozoa is yet to be explored. Simultaneously, these receptors in spermatozoa have opened new horizons to study molecular basis of cannabinoid induced infertility or subfertility and this can be managed employing suitable drugs. Furthermore, studies are also required to understand that how endocannabinoids regulate sperm function in terms of regulation of sperm fertility.

\section{ACKNOWLEDGEMENTS}

Authors also thank the Vice Chancellor, U.P. Pt. Deen Dayal Upadhyaya Pashu Chikitsa Vigyan Vishwavidyalaya, Mathura-281001. We are highly thankful to the Department of Biotechnology, Govt. of India (GOI).

of India for providing financial support (BT/PR27446/ AAQ/1/717/2018).

\section{CONFLICT OF INTEREST}

The authors declare no conflict of interest with any people or organizations in any financial or personal relationship.

\section{ABBREVIATIONS}

AA- Arachidonic acid 
AC-Adenyl cyclase

AR-Acrosome reaction

ABHD2- Alpha/beta hydrolase domain containing protein 2

AEA- Anandamide (N-Arachidonoylethanolamine)

AMT- Anandamide Membrane Transporter

2-AG- 2-Arachidonoyl glycerol

cAMP- cyclic Adenosine monophospohate

CB1- Cannabinoid receptor 1

CB2- Cannabinoid receptor 2

cPLA $_{2}$-Cytosolic Phospholipase $\mathrm{A}_{2}$

DAGL- Diacylglycerol lipase

eCBs- Endocannabinoids

ECS- Endocannabinoid system

EMT- Endocannabinoid Membrane Transporter

Et- $\mathrm{NH}_{2}-$ Ethanol amine

FAAH- Fatty acid amide hydrolase

FAK- Focal Adhesion Kinase

FSH- Follicle stimulating hormone

GPCR- G-protein coupled receptors

iNOS- inducible nitric oxide synthase,

LH- Luteinizing hormone

MAGL- Monoacylglycerol lipase

MAPK- Mitogen Activated Protein Kinase

NAPE-PLD- N-Acetylphosphatidyl ethanolamine hydrolysing phospholipase D

PPARs- Peroxisome proliferator activated receptor

THC- $\Delta$ 9-tetrahydrocannabinol

TRPV1- Transient receptor potential vanilloid type 1

ZP- Zona pellucida

\section{REFERENCES}

Agirregoitia, E., Carracedo, A., Subirán, N., Valdivia, A., Agirregoitia, N., Peralta, L., Velasco, G. and Irazusta, J.
2009. The CB2 cannabinoid receptor regulates human sperm cell motility. Fertil. Steril., 93(5): 1378- 1387.

Barbonetti, A., Vassalo, M.R.C., Fortunato, D., Francavilla, S., and Maccarone, M. 2010. Energetic metabolism and human sperm motility: impact of $\mathrm{CB} 1$ receptor activation. Endocrinology, 151(12): 5882-5892.

Battista, N., Bari, M. and Maccarrone, M. 2015. Endocannabinoids and reproductive events in health and disease. Endocannabinoids, 341-365

Burstein, S., Young, J.K. and Wright, G.E. 1995. Relationships between eicosanoids and cannabinoids. Are eicosanoids cannabimimetic agents? Biochem. Pharmacol., 50: 17351742.

Devane, W.A., Hanus, L., Breuer, A., Pertwee, R.G., Stevenson, L.A., Griffin, G., Gibson, D., Mandelbaum, A., Etinger, A. and Mechoulam, R. 1992. Isolation and structure of a brain constituent that binds to the cannabinoid receptor. Science, 258: 1946-1949.

Francavilla, F., Battista, N., Barbonetti, A., Vassalo, M.R.C., Rapino, C., Antonangelo, C., Pasquariello, N., Catanzaro, G., Barboni, B. and Maccarrone, M. 2009. Characterization of the endocannabinoid system in human spermatozoa and involvement of transient receptor potential vanilloid 1 receptor in their fertilizing ability. Endocrinology, 150(10): 4692-4700.

Gervasi, M.G., Rapanelli, M., Ribeiro, M.L., Farina, M., Billi, S., Franchi, A.M. and Martinez, S.P. 2009. The endocannabinoid system in bull sperm and bovine oviductal epithelium: role of anandamide in sperm-oviduct interaction. Reproduction, 137: 403-414.

Matsuda, L.A., Lolait, S.J., Brownstein, M.J., Young, A.C., and Bonner, T.I. 1990. Structure of a cannabinoid receptor and functional expression of the cloned cDNA. Nature, 346: 561-564.

Maccarrone, M., Lorenzon, T., Bari, M., Melino, G. and FinazziAgrò, A. 2000. Anandamide induces apoptosis in human cells via vanilloid receptors. Evidence for a protective role of cannabinoid receptors. J. Biol. Chem., 275: 31938-31945.

Maccarrone, M., Cecconi, S., Rossi, G., Battista, N., Pauselli, R. and Finazzi-Agrò, A. 2003. Anandamide activity and degradation are regulated by early postnatal ageing and follicle-stimulating hormone in mouse Sertoli cells. Endocrinology, 144: 20-28.

Maccarrone, M., Barboni, B., Paradisi, A., Bernabo, N., Gasperi, V., Pistilli, M.G., Fezza, F., Lucidi, P. and Mattioli, M. 2005. Characterization of the endocannabinoid system in boar spermatozoa and implications for sperm capacitation and acrosome reaction. J. Cell. Sci., 118: 4393-4404. 
Maccarrone, M. 2009. Endocannabinoids: friends and foes of reproduction. A review. Prog. Lip. Res., 48: 344-354.

Meccariello, R., Battista, N., Bradshaw, H.B. and Wang, H. 2014. Updates in reproduction coming from the endocannabinoid system. Int. J. Endocrinol., 412354 (https:// doi.org/10.1155/2014/412354).

Mechoulam, R., Ben-Shabat, S., Hanus, L., Ligumsky, M., Kaminski, N.E., Schatz, A.R., Gopher, A., Almog, S., Martin, B.R. and Compton, D.R. 1995. Identification of an endogenous 2-monoglyceride, present in canine gut, that binds to cannabinoid receptors. Biochem. Pharmacol., 50: 83-90.

Miller, M.R., Mannowetz, N., Iavarone, A.T., Safavi, R., Gracheva, E.O., Smith, J.F., Hill, R.Z., Bautista, D.M., Kirichok, Y. and Lishko, P.V. 2016. Unconventional endocannabinoid signaling governs sperm activation via sex hormone progesterone. Science, 352(6285): 555-559

Munro, S., Thomas, K.L. and Abu-Shaar, M. 1993. Molecular characterization of a peripheral receptor for cannabinoids. Nature, 365(6441): 61-65.

Pertwee R.G., Howlett A.C., Abood M.E., Alexander S.P.H., Di Marzo V., Elphick M.R., Greasley P.J., Hansen H.S., Kunos G., Mackie K., Mechoulam R., and Ross R. A. 2010. International Union of Basic and Clinical Pharmacology. LXXIX. Cannabinoid receptors and their ligands: beyond CB1 and CB2. Pharmacol. Rev., 62(4): 588-631.

Rapino, C., Battista, N., Bari, M. and Maccarrone, M. 2014. Endocannabinoids as biomarkers of human reproduction. Hum. Reprod. Update., 20(4): 501-516.

Ricci, G., Cacciola, G., Altucci, L., Meccariello, R., Pierantoni, R., Fasano, S. and Cobellis, G. 2007. Endocannabinoid control of sperm motility: The role of epididymus. Gen. Comp. Endocr., 153: 320-322.
Rossato M., Ion Popa F., Ferigo M., Clari G., and Foresta C. 2004, Human sperm express cannabinoid receptor CB1, the activation of which inhibits motility, acrosome reaction, and mitochondrial function. J. Clin. Endocr. Metab., 90(2): 984 991.

Rossi, G., Gasper, V., Paro, R., Barsacchi, D., Cecconi, S. and Maccarone, M. 2007. Follicle stimulating hormone activates fatty acid amide hydrolase by protein kinase $\mathrm{A}$ and aromatase- dependent pathways in mouse primary sertoli cells. Endocrinology, 148: 1431-1439.

Schmid, H.H.O. 2000. Pathways and mechanisms of $\mathrm{N}$-acylethanolamine biosynthesis: Can anandamide be generated selectively? Chem. Phys. Lipids., 108: 71-87.

Schuel, H., Burkman, L.J., Lippes, J., Crickard, K., Mahony, M.C., Giuffrida, A., Picone, R.P. and Makriyannis, A. 2002. Evidence that anandamide-signaling regulates human sperm functions required for fertilization. Mol. Repro. Dev., 63 : 376-387

Schule, H. 2006. Tuning the oviduct to the anandamide tone. $J$. Clin. Invest., 116(8): 2087-2090.

Sugiura, T., Kondo, S., Sukagawa, A., Nakane, S., Shinoda, A., Itoh, K., Yamashita, A. and Waku, K. 1995. 2-Arachidonoylglycerol: a possible endogenous cannabinoid receptor ligand in brain. Biochem. Bioph. Res. Co., 215: 8997.

Sun, X., Wang, H., Okabe, M., Mackie, K., Kingsley, P.J., Marnett L.J., Caravatt B.F. and Dey, S.K. 2009. Genetic loss of faah compromises male fertility in mice. Biol. Reprod., 80: $235-242$.

Van der Stelt, M. and Di Marzo, V. 2005. Anandamide as an intracellular messenger regulating ion channel activity. Prostag. Oth. Lipid. M., 77: 111-122. 
